\title{
MHD Flow due to the Nonlinear Stretching of a Porous Sheet
}

\author{
Tarek M. A. El-Mistikawy \\ Department of Engineering Mathematics \& Physics, Faculty of Engineering, Cairo University, Giza 11221, Egypt
}

Correspondence should be addressed to Tarek M. A. El-Mistikawy; t.mistik@gmail.com

Received 13 September 2015; Accepted 20 December 2015

Academic Editor: Luigi C. Berselli

Copyright (C) 2016 Tarek M. A. El-Mistikawy. This is an open access article distributed under the Creative Commons Attribution License, which permits unrestricted use, distribution, and reproduction in any medium, provided the original work is properly cited.

\begin{abstract}
The MHD flow due to the nonlinear stretching of a porous sheet is investigated. A closed form solution is obtained when the stretching rate is inversely proportional to the distance from the origin. Otherwise a uniformly valid asymptotic expansion, for large magnetic interaction number $\beta \sim \infty$, is developed. It coincides with a homotopy perturbation expansion for the problem. The asymptotic/homotopy perturbation expansion gives results in excellent agreement with accurate numerical results, for large as well as small values of $\beta$. For large $\beta$, the expansion, being asymptotic, needs a small number of terms, regardless of the mass transfer rate or the degree of nonlinearity. For small $\beta$, the expansion is a homotopy perturbation one. It needs considerably increasing number of terms with higher injection rates and/or with stretching rates approaching the inverse proportionality. It may even fail.
\end{abstract}

\section{Introduction}

The problem of the MHD flow due to the nonlinear stretching of a nonporous sheet was first formulated and analyzed by Chiam [1]. He chose a special magnetic field that allowed the problem to be cast in a self-similar form. He also explored the asymptotic solution for large magnetic interaction number $\beta$ and obtained a three-term expansion for the stream function that was marred by the presence of secular terms.

The problem has recently come under extensive study. The aim has been to construct approximate expansion solutions through newly developed analytical methods, with the help of computer symbolic manipulation software facilities. Hayat et al. [2] has used Adomian's decomposition method to construct an expansion in powers of the similarity coordinate $\eta$. Yildirim and Sezer [3], Fathizadeh et al. [4], and Sushila et al. [5] have arrived at the same power expansion through variants of the homotopy perturbation method. The power expansion has given accurate results only at a narrow range of $\eta$. This range has been extended through the use of Padé approximants in $[2,3]$.

Raftari et al. [6] have constructed a different homotopy problem, on which they have imposed an unnecessary restriction, and have obtained a two-term homotopy perturbation expansion involving secular terms.
In this paper, the problem of the MHD flow due to the nonlinear stretching, according to a power law of index $\alpha$, of a porous sheet is studied. When $\alpha=-1$, a closed form solution is obtained. When $\alpha \neq-1$, a uniformly valid asymptotic expansion as $\beta \sim \infty$ is obtained and is shown to be identical to a homotopy perturbation expansion of the problem. The present asymptotic/homotopy perturbation expansion alleviates problems facing previous works on Chiam's problem [1-6]. Consequences of the expansion being asymptotic or a homotopy perturbation one are assessed with regard to the number of terms retained, and the accuracy achieved.

\section{Formulation of the Problem}

An electrically conducting fluid is driven by a symmetrically stretching insulated sheet, lying along the $x$-axis. The stretching rate is proportional to $|x|^{\alpha}$. The sheet is porous allowing fluid flow of rate proportional to $|x|^{(\alpha-1) / 2}$ in the normal $y$-direction, along which a magnetic field of strength proportional to $|x|^{(\alpha-1) / 2}$ is applied. The power law index $\alpha$ may be positive or negative. 
For $x>0$, the boundary layer equations write [1]

$$
\begin{gathered}
\frac{\partial u}{\partial x}+\frac{\partial v}{\partial y}=0 \\
u \frac{\partial u}{\partial x}+v \frac{\partial u}{\partial y}=\nu \frac{\partial^{2} u}{\partial y^{2}}-\frac{\sigma B^{2} x^{\alpha-1}}{\rho} u
\end{gathered}
$$

with the boundary conditions

$$
\begin{aligned}
& y=0: u=\omega x^{\alpha}, \\
& v=-\Gamma x^{(\alpha-1) / 2}, \\
& y \longrightarrow \infty: u \longrightarrow 0 .
\end{aligned}
$$

$(u, v)$ are the velocity components in the $(x, y)$ directions, $(\omega>0, \Gamma, B)$ are (stretching, suction, and magnetic field) coefficients, $(\rho, \nu, \sigma)$ are the fluid (density, kinematic viscosity, and electric conductivity), respectively.

The following self-similar problems can be formulated, with $\psi$ denoting the stream function.

\section{Case of $\alpha=-1$}

We introduce the following similarity variables and parameters:

$$
\begin{aligned}
& \eta=x^{-1} \sqrt{\frac{\omega}{v}} y, \\
& \psi=\sqrt{\nu \omega} f(\eta), \\
& u=\omega x^{-1} \frac{d f}{d \eta}, \\
& v=\sqrt{\nu \omega} x^{-1} \eta \frac{d f}{d \eta}, \\
& \Gamma=0, \\
& \beta=\frac{\sigma B^{2}}{\rho \omega} .
\end{aligned}
$$

The problem takes the form

$$
\begin{aligned}
\frac{d^{3} f}{d \eta^{3}}+\left(\frac{d f}{d \eta}\right)^{2}-\beta \frac{d f}{d \eta} & =0, \\
\frac{d f}{d \eta}(0) & =1, \\
\frac{d f}{d \eta}(\infty) & =0, \\
f(0) & =0 .
\end{aligned}
$$

The last condition corresponds to $\psi=0$ at the sheet.
The problem admits the following solution:

$$
\begin{aligned}
& f=6 \beta^{1 / 2} b^{2}\left(\frac{1}{b^{2}+(2 / 3 \beta) e^{-\beta^{1 / 2} \eta}}-\frac{1}{b^{2}+2 / 3 \beta}\right), \\
& b=1+\sqrt{1-\frac{2}{3 \beta}} .
\end{aligned}
$$

Thus, no such self-similar solution is possible, when $\beta<2 / 3$. Differentiation gives

$$
\begin{aligned}
\frac{d f}{d \eta} & =\frac{4 b^{2} e^{-\beta^{1 / 2} \eta}}{\left(b^{2}+(2 / 3 \beta) e^{-\beta^{1 / 2} \eta}\right)^{2}}, \\
\frac{d^{2} f}{d \eta^{2}} & =\frac{-4 b^{2} \beta^{1 / 2} e^{-\beta^{1 / 2} \eta}\left(b^{2}-(2 / 3 \beta) e^{-\beta^{1 / 2} \eta}\right)}{\left(b^{2}+(2 / 3 \beta) e^{-\beta^{1 / 2} \eta}\right)^{3}} .
\end{aligned}
$$

The surface shear is represented by

$$
\frac{d^{2} f}{d \eta^{2}}(0)=-\frac{1}{2} \beta^{1 / 2} b\left(1-\frac{2}{3 \beta b^{2}}\right) .
$$

The flow is of a source type. The stream lines are lines of constant $\eta$, that is, rays emanating from the origin. The source strength measured by the rate of fluid discharge (to the first quadrant) is represented by

$$
f(\infty)=\frac{2}{\beta^{1 / 2} b} .
$$

This discharge must come from a slit at the origin.

\section{Case of $\alpha \neq-1$}

We introduce the following similarity variables and parameters:

$$
\begin{aligned}
& \eta=\sqrt{\frac{|\alpha+1| \omega}{2 \nu} x^{(\alpha-1) / 2} y,} \\
& \psi=\sqrt{\frac{2 \nu \omega}{|\alpha+1|} x^{(\alpha-1) / 2} f(\eta),} \\
& u=\omega x^{\alpha} \frac{d f}{d \eta}, \\
& \nu=-\Gamma x^{(\alpha-1) / 2}\left(f+\frac{\alpha-1}{\alpha+1} \eta \frac{d f}{d \eta}\right), \\
& \Gamma=\gamma \frac{\alpha+1}{2} \sqrt{\frac{2 \nu \omega}{|\alpha+1|}}, \\
& \beta=\frac{2 \sigma B_{0}^{2}}{|\alpha+1| \rho \omega}, \\
& \lambda=\frac{2 \alpha}{1+\alpha}, \\
& \delta=\operatorname{sgn}(\alpha+1) .
\end{aligned}
$$


The problem takes the form

$$
\begin{aligned}
\frac{d^{3} f}{d \eta^{3}}+\delta\left[f \frac{d^{2} f}{d \eta^{2}}-\lambda\left(\frac{d f}{d \eta}\right)^{2}\right]-\beta \frac{d f}{d \eta} & =0, \\
\frac{d f}{d \eta}(0) & =1, \\
\frac{d f}{d \eta}(\infty) & =0, \\
f(0) & =\gamma .
\end{aligned}
$$

Chiam's nonporous sheet problem [1] corresponds to $\gamma=0$ and $\delta=+1$.

The case of $\lambda=1(\alpha=1$ and $\delta=+1)$ admits the exact solution

$$
f=\gamma+\frac{\left(1-e^{-c \eta}\right)}{c}
$$

where $c$ is the positive root of the quadratic equation

$$
c^{2}-\gamma c-(1+\beta)=0
$$

This solution was obtained by Chakrabarti and Gupta [7]. It degenerates to that of Andersson [8] for a nonporous sheet $\gamma=0$, to that of P. S. Gupta and A. S. Gupta [9] in the absence of the magnetic field $\beta=0$, and to that of Crane [10] when $\beta=\gamma=0$.

4.1. Asymptotic Limit as $\beta \sim \infty$. We are interested in the limiting behavior of the flow as the magnetic interaction number $\beta$ grows indefinitely. In the limit as $\beta \sim \infty, f=O(1)$ having the fixed value $\gamma$ at $\eta=0$. The electromagnetic force term $\beta(d f / d \eta)$ is, thus, of leading order. It can be balanced by the viscous term $d^{3} f / d \eta^{3}$ in a contracting region in which $\eta=O\left(\beta^{-1 / 2}\right)$.

Introducing the stretched variable $\zeta=\beta^{1 / 2} \eta$, the problem becomes

$$
\begin{aligned}
f^{\prime \prime \prime}-f^{\prime} & =\beta^{-1 / 2} \delta\left(\lambda f^{\prime 2}-f f^{\prime \prime}\right), \\
f(0) & =\gamma, \\
f^{\prime}(0) & =\beta^{-1 / 2}, \\
f^{\prime}(\infty) & =0
\end{aligned}
$$

where primes denote differentiation with respect to $\zeta$.

The straightforward expansions of $f$ would involve secular terms. To overcome this difficulty we follow the lead of $\mathrm{He}$ [11] and strain the coefficient 1 of $f^{\prime}$. To put this in effect, we rewrite (8a) as

$$
\begin{aligned}
f^{\prime \prime \prime}-a^{2} f^{\prime} & =\beta^{-1 / 2} \delta\left(\lambda f^{\prime 2}-f f^{\prime \prime}\right), \\
a^{2} & =1 .
\end{aligned}
$$

Introducing the asymptotic expansions

$$
\begin{aligned}
& f \sim \sum_{k=0} \beta^{-k / 2} f_{k}, \\
& a \sim \sum_{k=0} \beta^{-k / 2} a_{k}
\end{aligned}
$$

and equating like powers of $\beta^{-1 / 2}$, we arrive at problems for $f_{k}, k \geq 0$. The problem for $f_{0}$ is

$$
\begin{array}{r}
f_{0}^{\prime \prime \prime}-a_{0}^{2} f_{0}^{\prime}=0, \\
f_{0}(0)=\gamma, \\
f_{0}^{\prime}(0)=0, \\
f_{0}^{\prime}(\infty)=0
\end{array}
$$

with the solution

$$
f_{0}=\gamma
$$

The problem for $f_{1}$ is, then,

$$
\begin{array}{r}
f_{1}^{\prime \prime \prime}-a_{0}^{2} f_{1}^{\prime}=0, \\
f_{1}(0)=0, \\
f_{1}^{\prime}(0)=1, \\
f_{1}^{\prime}(\infty)=0
\end{array}
$$

with the solution

$$
\begin{aligned}
& f_{1}=\frac{(1-E)}{a_{0}}, \\
& E=e^{-a_{0} \zeta}
\end{aligned}
$$

The problem for $f_{2}$ is

$$
\begin{aligned}
f_{2}^{\prime \prime \prime}-a_{0}^{2} f_{2}^{\prime} & =\left(\delta \gamma a_{0}+2 a_{0} a_{1}\right) E \\
f_{2}(0) & =0 \\
f_{2}^{\prime}(0) & =0 \\
f_{2}^{\prime}(\infty) & =0 .
\end{aligned}
$$

The right hand side of (15a) produces a secular term, the removal of which requires the regularity condition

$$
\delta \gamma a_{0}+\sum_{j=0}^{1} a_{j} a_{1-j}=0
$$

leading to the solution

$$
f_{2}=0 \text {. }
$$

Likewise, the problem for $f_{3}$ gives the regularity condition

$$
\delta+\sum_{j=0}^{2} a_{j} a_{2-j}=0
$$


and the solution

$$
f_{3}=\frac{\delta(1-\lambda)\left(1-2 E+E^{2}\right)}{6 a_{0}^{3}} .
$$

The problem for $f_{k}, k \geq 2$, is

$$
\begin{aligned}
& f_{k}^{\prime \prime \prime}-a_{0}^{2} f_{k}^{\prime} \\
& =\sum_{i=0}^{k-1}\left[\delta\left(\lambda f_{i}^{\prime} f_{k-1-i}^{\prime}-f_{i} f_{k-1-i}^{\prime \prime}\right)+f_{i}^{\prime} \sum_{j=0}^{k-i} a_{j} a_{k-i-j}\right], \\
& f_{k}(0)=0, \\
& f_{k}^{\prime}(0)=0, \\
& f_{k}^{\prime}(\infty)=0 .
\end{aligned}
$$

In general, the solution for $f_{k}, k \geq 0$, is of the form

$$
\begin{aligned}
f_{k} & =\sum_{n=0}^{(k+1) \mid 2} f_{k n} E^{n}, \\
(k+1) \mid 2 & =\operatorname{int}\left[\frac{(k+1)}{2}\right] .
\end{aligned}
$$

Expressions to determine $f_{k n}$ 's are given in Appendix. It is noted that, of all $a_{k}$ 's, only $a_{0}$ appears in the expressions for $f_{k n}$ 's. Moreover, the following properties of $f_{k n}$ 's can be proved by induction:

(a) for $k \geq 3, f_{k n}$ has $(1-\lambda)$ as a factor,

(b) for even $k \geq 0, f_{k n}$ has $\gamma$ as a factor,

(c) for $k \geq 0, f_{k n}$ is inversely proportional to $a_{0}^{k}$; that is,

$$
f_{k n}=\frac{h_{k n}}{a_{0}^{k}},
$$

where $h_{k n}$ 's are given by the same expressions in Appendix for $f_{k n}$ 's but with $a_{0}$ set equal to unity.

For $k \geq 1$, the regularity condition is

$$
\delta a_{0} f_{k-10}+\sum_{j=0}^{k} a_{j} a_{k-j}=0,
$$

which combines with (9b) and (10b) to give the following equation for $a_{0}$ :

$$
a_{0}^{2}-\delta a_{0} \sum_{k=1} \beta^{-k / 2} f_{k-10}-1=0 .
$$

In the case of $\lambda=1(\alpha=1 \Rightarrow \delta=+1), f_{k}=0$ for $k \geq 2$ due to property (a) of $f_{k n}$. Therefore,

$$
\begin{aligned}
f & =\gamma+\frac{\beta^{-1 / 2}(1-E)}{a_{0}}, \\
\beta a_{0}^{2}-\gamma \beta^{1 / 2} a_{0}-(1+\beta) & =0 .
\end{aligned}
$$

Equations (23a) and (23b) are identical to the exact solution expressed by (7a) and (7b), as we set $\beta^{1 / 2} a_{0}=c$.
4.2. Homotopy Perturbation Solution. We now seek a homotopy perturbation solution to the problem described by (8a), $(8 b),(8 c)$, and (8d). To that end, we formulate an associated homotopy problem

$$
\begin{aligned}
f^{\prime \prime \prime}-f^{\prime} & =p \beta^{-1 / 2} \delta\left(\lambda f^{\prime 2}-f f^{\prime \prime}\right), \\
f(0) & =\gamma, \\
f^{\prime}(0) & =p \beta^{-1 / 2}, \\
f^{\prime}(\infty) & =0,
\end{aligned}
$$

where $p$ is the embedding parameter, varying from 0 to 1 .

Setting $p \beta^{-1 / 2}=\bar{\beta}^{-1 / 2}$, the homotopy problem (24a), (24b), (24c), and (24d) acquires the same form as the physical problem (8a), (8b), (8c), and (8d) and coincides with it at the final step of the homotopy perturbation procedure, when we set $p=1$.

As both problems are treated as perturbation problems, the resulting expansions will be identical.

The present homotopy perturbation expansion is free from secular terms and may involve as many terms as one chooses. Allowing a secular term, Raftari et al. [6] obtained a two-term homotopy perturbation expansion, for the case of $\gamma=0$, that imposed a restriction on $\lambda$ and $\beta$, namely, $3 \beta+2 \lambda+1>0$, and that could not be extended to include further terms.

Through variants of the homotopy perturbation method, Yildirim and Sezer [3], Fathizadeh et al. [4], and Sushila et al. [5] obtained the same polynomial solution to the case of $\gamma=0$ that had been obtained earlier by Hayat et al. [2] via Adomian's decomposition method. Results presented in $[4,5]$ showed deviation from numerically calculated results, beyond a narrow range of $\eta$. This range was extended through the use of Padé approximants in [2, 3]. In contrast, the present homotopy perturbation solution, involving negative exponentials, is valid for all values of $\eta \in[0, \infty)$.

Keeping enough terms of the present homotopy perturbation expansion, it is expected to get accurate results for all values of $\beta$. However, the expansion, being an asymptotic one as $\beta \sim \infty$ as well, should require few terms to give accurate results for large $\beta$.

\section{Numerical Procedures}

To put the above expansions into use, we cut short the summations in (10a) and (21) to the same finite upper limit $K>0$. The following iterative procedure is then adopted.

(1) For the given $\lambda$ and $\gamma$, determine all $h_{k n}$ 's using the formulas in Appendix.

(2) Choose a starting value for $a_{0}$.

(3) Calculate $f_{k n}$ 's using (20).

(4) Introduce $f_{k 0}$ 's into (22) and solve it for a new $a_{0}$. 
(5) If convergence of $a_{0}$ is not reached, that is, $\mid a_{0}^{\text {new }}-$ $a_{0}^{\text {old }} \mid \geq 10^{-10}$, go to step (3).

(6) Otherwise, evaluate $f$ and its derivatives for the given $\beta$.

Using (20), we can transfer (22) to an algebraic equation of degree $K$ in $a_{0}$ and can derive a Newton iterative formula for $a_{0}$ to be used in step (4); namely,

$$
a_{0}^{\text {new }}=a_{0}^{\text {old }}-\frac{\left(a_{0}^{\text {old }}\right)^{2}-\delta\left(a_{0}^{\text {old }}\right) \sum_{k=1}^{K} \beta^{-k / 2} f_{k-10}-1}{2\left(a_{0}^{\text {old }}\right)-\delta \sum_{k=1}^{K} \beta^{-k / 2}(2-k) f_{k-10}}
$$

The two quantities of physical significance are the surface shear represented by

$$
\frac{d^{2} f}{d \eta^{2}}(0)=\beta a_{0}^{2} \sum_{k=0}^{K} \beta^{-k / 2} \sum_{n=1}^{(k+1) \mid 2} n^{2} f_{k n}
$$

and the rate of flow entrainment represented by

$$
f(\infty)=\sum_{k=0}^{K} \beta^{-k / 2} f_{k 0}
$$

These two quantities, together with $a_{0}$, will be the focus of our calculations.

The results of the abovementioned procedure are tested against corresponding numerical results. The problem described by (6a), (6b), (6c), and (6d) is solved numerically using Keller's two-point, second-order-accurate, finitedifference scheme [12]. A uniform step size $\Delta \eta=0.01$ is used on a finite domain $0 \leq \eta \leq \eta_{\infty}$. The value of $\eta_{\infty}=70$ is chosen sufficiently large in order to insure the asymptotic satisfaction of the far-field condition (6d). The nonlinear terms are quasi-linearized, and an iterative procedure is implemented, terminating when the maximum error in $\left(d^{2} f / d \eta^{2}\right)(0)$ and $f\left(\eta_{\infty}\right)$ becomes less than $10^{-10}$.

The accuracy of the numerical procedure was established by comparison with the exact solution for $\lambda=1$ with different combinations of $\gamma$ and $\beta$, both producing identical results to, at least, the tenth significant figure.

The numerical solution calculates $a_{0}$ as the positive solution of the quadratic equation

$$
a_{0}^{2}-\delta a_{0} \beta^{-1 / 2} f\left(\eta_{\infty}\right)-1=0,
$$

which is the result of substitution of (26b) into (22). This guides the choice of a suitable starting value for $a_{0}$, in step (2) of the iterative procedure described above.

\section{Results}

In the results presented below for the expansion solutions, two factors are of concern: the choice of the cutoff number $K$ and the accuracy in comparison to the numerical solutions.

First, it should be noted that $K$ has to be $\geq 3$ for the effect of $\lambda$ to be retained. As $K$ increases, progressive "saturation" in the significant figures of $a_{0}$ is achieved. Where to stop depends on the flow parameters $\beta, \lambda$, and $\gamma$. To illustrate
TABLE 1: Dependence of $K$ on $\beta[\lambda=1.5(\alpha=3), \gamma=0]$.

\begin{tabular}{ccccc}
\hline$\beta$ & $K$ & $a_{0}$ & $\frac{d^{2} f}{d \eta^{2}}(0)$ & $f\left(\eta_{\infty}\right)$ \\
\hline \multirow{4}{*}{100} & 5 & 1.004983457 & -10.06643912 & 0.09942195793 \\
& 9 & 1.004983454 & -10.06643909 & 0.09942195832 \\
& Numerical & 1.004983447 & -10.06642517 & 0.09942182036 \\
\hline & 9 & 1.398448416 & -1.525271655 & 0.6833820804 \\
1 & 11 & 1.398454500 & -1.525274499 & 0.6833795495 \\
& 13 & 1.398454580 & -1.525274777 & 0.6833794078 \\
& Numerical & 1.398453909 & -1.525273038 & 0.6833785009 \\
\hline \multirow{4}{*}{0.01} & 19 & 9.546679245 & -1.153004023 & 0.9441932777 \\
& 21 & 9.546680372 & -1.153004063 & 0.9441931944 \\
& 23 & 9.546680386 & -1.153004075 & 0.9441931820 \\
& Numerical & 9.546668853 & -1.153003010 & 0.9441920274 \\
\hline
\end{tabular}

the dependence on $\beta$, consider the case of $\lambda=1.5(\alpha=3), \gamma=$ 0 . Table 1 presents results at consecutive $K$ 's for $\beta=100,1$, and 0.01 . (We proceed in odd values of $K$, to avoid programming complexities.) It is noted that, for the larger $\beta$, saturation in 6 significant figures is attained at $K=5$. The intermediate and smaller $\beta$ 's achieve the same level of saturation at $K=11$ and 21 , respectively.

Also presented in Table 1 are the corresponding numerical results. As expected, for the larger $\beta$, few terms of the expansion, which is asymptotic, give excellent agreement with the numerical solution. For the smaller $\beta$ 's, the expansion is no longer asymptotic but should be considered a homotopy perturbation one. Its agreement with the numerical solution is as excellent.

Does this agreement extend to other values of $\gamma$ and $\lambda$ ? The answer is given in Tables 2, 3, and 4, in which, for each indicated value of $\gamma$ or $\lambda$, the first line gives the expansion solution and, if possible, the value of $K$ for saturation in 6 significant figures, while the second line gives the numerical solution.

Table 2 includes cases of suction $\gamma>0$ and injection $\gamma<0$. Agreement with the numerical solutions to at least five significant figures is observed. At the larger $\beta$, the asymptotic nature of the expansions allows a fixed small value of $K$, regardless of the value of $\gamma$. At the smaller $\beta$ 's, the homotopy perturbation expansions are aided by suction and progressively hurt by injection, to the extent that no solution is possible as indicated by the void entries. Either saturation in enough significant figures could not be attained, or convergence of the iterative process for $a_{0}$ could not be reached. As an illustration, consider the case of $\beta=1.0$ and $\gamma=-2$. The following are some $a_{0}\{K\}$ results that show no tendency toward saturation: $0.7282\{5\}, 0.7291\{7\}, 0.7254\{9\}$, $0.716\{11\}, 0.700\{13\}, \ldots, 0.687\{19\}, 0.723\{21\}, 0.736\{23\}$, $0.741\{25\}, 0.737\{27\}, 0.714\{29\}, \ldots, 0.749\{39\}, 0.75914\{41\}$, $0.75953\{43\}, 0.747\{45\}, \ldots, 0.756\{55\}, 0.7723\{57\}, 0.7757\{59\}$, $0.7717\{61\}, \ldots, 0.780\{73\}$. The ellipses indicate $K$-ranges of no convergence to a positive $a_{0}$. When $K=35,51$, and $62, a_{0}$ converges to the same negative value -2.7277089043 . 
TABLE 2: Validity of the expansion solution at different values of $\beta$ and $\gamma, \lambda=1.5(\alpha=3)$.

\begin{tabular}{|c|c|c|c|c|c|c|c|c|c|}
\hline$\beta$ & & 100 & & & 1.0 & & & 0.01 & \\
\hline$\gamma$ & $\frac{d^{2} f}{d \eta^{2}}(0)$ & $K$ & $f\left(\eta_{\infty}\right)$ & $\frac{d^{2} f}{d \eta^{2}}(0)$ & $K$ & $f\left(\eta_{\infty}\right)$ & $\frac{d^{2} f}{d \eta^{2}}(0)$ & $K$ & $f\left(\eta_{\infty}\right)$ \\
\hline-2 & -9.11655 & 5 & -1.89021 & - & & - & - & & - \\
\hline 2 & -9.11654 & & -1.89021 & -0.844839 & & -0.714243 & -0.558696 & & 0.249190 \\
\hline-1 & -9.57913 & 5 & -0.895514 & -1.11742 & 29 & -0.0471147 & - & & - \\
\hline 1 & -9.57911 & & -0.895515 & -1.11742 & & -0.0471154 & -0.778042 & & 0.504566 \\
\hline 0 & -10.0664 & 5 & 0.0994220 & -1.52527 & 13 & 0.683379 & -1.15300 & 17 & 0.944193 \\
\hline & -10.0664 & & 0.0994218 & -1.52527 & & 0.683379 & -1.15300 & & 0.944192 \\
\hline 1 & -10.5786 & 5 & 1.09460 & -2.09551 & 9 & 1.48920 & -1.74219 & 11 & 1.59722 \\
\hline & -10.5786 & & 1.09460 & -2.09551 & & 1.48920 & -1.74219 & & 1.59721 \\
\hline 2 & -11.1155 & 5 & 2.09003 & -2.81024 & 11 & 2.36110 & -2.50877 & 11 & 2.40646 \\
\hline 2 & -11.1154 & & 2.09003 & -2.81023 & & 2.36110 & -2.50877 & & 2.40646 \\
\hline
\end{tabular}

TABLE 3: Validity of the expansion solution at different values of $\beta$ and $\lambda<2, \gamma=0$.

\begin{tabular}{|c|c|c|c|c|c|c|c|c|c|}
\hline \multirow{2}{*}{$\begin{array}{l}\beta \\
\lambda\end{array}$} & \multicolumn{3}{|c|}{100} & \multicolumn{3}{|c|}{1} & \multicolumn{3}{|c|}{0.01} \\
\hline & $\frac{d^{2} f}{d \eta^{2}}(0)$ & $K$ & $f\left(\eta_{\infty}\right)$ & $\frac{d^{2} f}{d \eta^{2}}(0)$ & K & $f\left(\eta_{\infty}\right)$ & $\frac{d^{2} f}{d \eta^{2}}(0)$ & $K$ & $f\left(\eta_{\infty}\right)$ \\
\hline \multirow{2}{*}{-1.5} & -9.96665 & 5 & 0.0999166 & -0.652972 & 269 & 0.912433 & - & & - \\
\hline & -9.96672 & & 0.0999173 & -0.652978 & & 0.912440 & 0.696751 & & 1.70859 \\
\hline \multirow{2}{*}{-1} & -9.98335 & 5 & 0.0998335 & -0.851110 & 113 & 0.851110 & - & & - \\
\hline & -9.98340 & & 0.0998340 & -0.851114 & & 0.851114 & -0.0140447 & & 1.40447 \\
\hline \multirow{2}{*}{-0.5} & -10.0000 & 5 & 0.0997506 & -1.01756 & 53 & 0.804077 & - & - & - \\
\hline & -10.0001 & & 0.0997510 & -1.01756 & & 0.804080 & -0.379674 & & 1.24358 \\
\hline \multirow{2}{*}{0} & -10.0167 & 5 & 0.0996680 & -1.16333 & 33 & 0.766078 & -0.634631 & 147 & 1.13644 \\
\hline & -10.0167 & & 0.0996683 & -1.16333 & & 0.766080 & -0.634630 & & 1.13645 \\
\hline \multirow{2}{*}{0.5} & -10.0333 & 5 & 0.0995857 & -1.29435 & 19 & 0.734312 & -0.835754 & 55 & 1.05725 \\
\hline & -10.0333 & & 0.0995859 & -1.29435 & & 0.734313 & -0.835755 & & 1.05725 \\
\hline \multirow{2}{*}{1} & -10.0499 & 3 & 0.0995037 & -1.41421 & 3 & 0.707107 & -1.004988 & 3 & 0.995037 \\
\hline & -10.0499 & & 0.0995037 & -1.41421 & & 0.707107 & -1.004988 & & 0.995037 \\
\hline \multirow{2}{*}{1.5} & -10.0664 & 5 & 0.0994220 & -1.52527 & 13 & 0.683379 & -1.15300 & 17 & 0.944193 \\
\hline & -10.0664 & & 0.0994218 & -1.52527 & & 0.683379 & -1.15300 & & 0.944192 \\
\hline
\end{tabular}

TABLE 4: Least possible $\beta$ (in whole numbers) for $\lambda>2, \gamma=0$.

\begin{tabular}{lllll}
\hline$\lambda$ & $\beta$ & $\frac{d^{2} f}{d \eta^{2}}(0)$ & $K$ & $f\left(\eta_{\infty}\right)$ \\
\hline 2.5 & \multirow{2}{*}{3} & -0.984756 & 17 & 0.850785 \\
& & -0.984762 & & 0.850788 \\
\hline \multirow{2}{*}{5} & 4 & -0.439138 & 69 & 0.990757 \\
& \multirow{2}{*}{7.5} & -0.439173 & & 0.990754 \\
\hline \multirow{2}{*}{10} & 6 & -0.742926 & 81 & 0.694277 \\
& \multirow{2}{*}{8} & -0.742974 & & 0.694285 \\
\hline
\end{tabular}

Otherwise, the left hand side of (22) drawn versus $a_{0}$, in the range indicated by the numerical solution, does not cross the zero line.
Table 3 includes cases of stretching sheets with progressively increasing rate $\alpha>0(0<\lambda<2)$ and progressively decreasing rate $-1<\alpha \leq 0(\lambda \leq 0)$, which are associated with $\delta=+1$. At the larger $\beta$, the asymptotic expansions allow a fixed small value of $K$, regardless of the value of $\lambda$. At the smaller $\beta$ 's, the homotopy perturbation expansions saturate readily when $\lambda>0$. However, as $\lambda$ decreases negatively they tend toward saturation with painful slowness, hence the void entries. As for accuracy, the expansion and numerical solutions are in excellent agreement, differing, at worst, by 1 in the fifth significant figure.

Other cases of progressively decreasing stretching rate $\alpha<-1(\lambda>2)$, which are associated with $\delta=-1$, are given in Table 4 . The indicated values of $\beta$ are the least whole numbers below which no solution was possible either numerically or through the expansions. As $\alpha$ gets closer to -1 ( $\lambda$ increases), the least $\beta$ 's get higher, the homotopy perturbation solutions require more terms for saturation, and their agreement with the numerical solutions deteriorates. 
(It should be noted that the results presented in [1-4] for the case of $\lambda=5$ are incorrect, as they were associated wrongly with $\delta=+1$.)

\section{Conclusion}

The problem of the MHD flow due to a nonlinearly stretching porous sheet has been investigated. A closed form solution has been obtained when the stretching rate is inversely proportional to distance (the power law index $\alpha=-1$ ). Otherwise, uniformly valid asymptotic expansion, for large magnetic interaction numbers $\beta \sim \infty$, has been developed. This expansion has been shown to coincide with a homotopy perturbation expansion for the problem. The expansion gives results in excellent agreement with accurate numerical results, for large as well as small values of $\beta$. For large $\beta$, the expansion being asymptotic, a small number of terms are needed regardless of the value of $\alpha$ or the suction rate $\gamma$. For small $\beta$, the expansion is a homotopy perturbation one. It needs considerably increasing number of terms as $\alpha$ or $\gamma$ grows negatively and may even fail.

The problem belongs to the class of problems identified below, whose asymptotic expansions and homotopy perturbation expansions are identical. The study helps realize the potential and limitations of asymptotic and homotopy perturbation methods.

Let $u$ be a sought after vector function which is defined on a domain $D$ with boundary $\Omega$ and governed by the following physical problem

$$
\begin{aligned}
L_{D}(u) & =\varepsilon R_{D}(u) \quad \text { in } D, \\
L_{\Omega}(u) & =\varepsilon R_{\Omega}(u) \\
\text { or } L_{\Omega}(u) & =R_{\Omega}(u)
\end{aligned}
$$

on $\Omega$,

where $\varepsilon$ is a small parameter and $L_{D}$ and $L_{\Omega}$ are left hand side operators, while $R_{D}$ and $R_{\Omega}$ are right hand side operators.

We form the associated homotopy problem

$$
\begin{aligned}
L_{D}(u) & =p \varepsilon R_{D}(u) \quad \text { in } D, \\
L_{\Omega}(u) & =p \varepsilon R_{\Omega}(u) \\
\text { or } L_{\Omega}(u) & =R_{\Omega}(u)
\end{aligned}
$$

on $\Omega$,

where $p$ is the embedding parameter, varying from 0 to 1 .

Setting $\bar{\varepsilon}=p \varepsilon$, the homotopy problem acquires the same form as the physical problem and coincides with it at the final step of the homotopy perturbation method, when we set $p=$ 1 .

As both problems are treated as perturbation problems, the resulting expansions will be identical.

\section{Appendix}

The following expressions determine the coefficients $f_{k n}$ in $f_{k}=\sum_{n=0}^{(k+1) \mid 2} f_{k n} E^{n}, k>3$. They involve summations, the limits of which may contain the forms $r \mid 2=\operatorname{int}(r / 2)$, $p \vee q=\min (p, q), p \wedge q=\max (p, q)$, se = even $s$, or $s o=$ odd $s$ where $p, q, r$, and $s$ are integers. When the upper limit is less than the lower limit, the summation is to be zeroed.

Given

$$
\begin{aligned}
& f_{00}=\gamma, \\
& f_{10}=-f_{11}=\frac{1}{a_{0}}, \\
& f_{20}=f_{21}=0, \\
& f_{30}=-\frac{1}{2} f_{31}=f_{32}=\frac{\delta(1-\lambda)}{6 a_{0}^{3}},
\end{aligned}
$$

then for even $k \geq 4$ and $n=2 \rightarrow k / 2$

$$
\begin{aligned}
\delta a_{0}\left(n^{3}-n\right) f_{k n} & n^{2} f_{00} f_{k-1 n}-n \sum_{i=2 n-1}^{k-1} f_{k-1-i 0} f_{i n} \\
& -\lambda \sum_{i=1}^{k-1} \sum_{m=1 \wedge n-(i+1) \mid 2}^{k|2-(i+1)| 2 \vee n-1}(n-m) m f_{i n-m} f_{k-1-i m} \\
& +\sum_{i=1}^{k-1} \sum_{m=1 \wedge n-(i+1) \mid 2}^{k|2-(i+1)| 2 \vee n} m^{2} f_{i n-m} f_{k-1-i m}
\end{aligned}
$$

and for odd $k \geq 5$ and $n=2 \rightarrow(k-1) / 2$

$$
\begin{aligned}
\delta a_{0} & \left(n^{3}-n\right) f_{k n}=n^{2} f_{00} f_{k-1 n}-n \sum_{i=2 n-1}^{k-1} f_{k-1-i 0} f_{i n} \\
& -\lambda\left[\sum_{i e=2}^{k-1} \sum_{m=1 \wedge n-i \mid 2}^{(k-1-i) \mid 2 \vee n-1}(n-m) m f_{i n-m} f_{k-1-i m}\right. \\
+ & \left.\sum_{i o=1}^{k-2} \sum_{m=1 \wedge n-(i+1) \mid 2}^{(k-i) \mid 2 \vee n-1}(n-m) m f_{i n-m} f_{k-1-i m}\right] \\
+ & \sum_{i e=2}^{k-1} \sum_{m=1 \wedge n-i \mid 2}^{(k-1-i) \mid 2 \vee n} m^{2} f_{i n-m} f_{k-1-i m} \\
+ & \sum_{i o=1}^{k-2} \sum_{m=1 \wedge n-(i+1) \mid 2}^{(k-i) \mid 2 \vee n} m^{2} f_{i n-m} f_{k-1-i m}
\end{aligned}
$$

with

$$
\begin{aligned}
\delta a_{0} & {\left[\left(\frac{k+1}{2}\right)^{3}-\left(\frac{k+1}{2}\right)\right] f_{k((k+1) / 2)} } \\
= & \sum_{i o=1}^{k-2}\left[\left(\frac{k-i}{2}\right)-\lambda\left(\frac{i+1}{2}\right)\right]\left(\frac{k-i}{2}\right) \\
\cdot & f_{i((i+1) / 2)} f_{k-1-i((k-i) / 2)} .
\end{aligned}
$$


The boundary conditions (18c) and then (18b) determine $f_{k 1}=-\sum_{n=2}^{(k+1) \mid 2} n f_{k n}$ and then $f_{k 0}=-\sum_{n=1}^{(k+1) \mid 2} f_{k n}$.

The expressions for $h_{k n}$ 's are identical to those for $f_{k n}$ 's but with $a_{0}=1$.

\section{Conflict of Interests}

The author declares that there is no conflict of interests regarding the publication of this paper.

\section{References}

[1] T. C. Chiam, "Hydromagnetic flow over a surface stretching with a power-law velocity," International Journal of Engineering Science, vol. 33, no. 3, pp. 429-435, 1995.

[2] T. Hayat, Q. Hussain, and T. Javed, "The modified decomposition method and Padé approximants for the MHD flow over a non-linear stretching sheet," Nonlinear Analysis: Real World Applications, vol. 10, no. 2, pp. 966-973, 2009.

[3] A. Yildirim and S. A. Sezer, "Non-perturbative solution of the magnetohydrodynamic flow over a nonlinear stretching sheet by homotopy perturbation method-padé technique," Zeitschrift für Naturforschung, vol. 65, no. 12, pp. 1106-1110, 2010.

[4] M. Fathizadeh, M. Madani, Y. Khan, N. Faraz, A. Yildirim, and S. Tutkun, "An effective modification of the homotopy perturbation method for MHD viscous flow over a stretching sheet," Journal of King Saud University-Science, vol. 25, no. 2, pp. 107-113, 2013.

[5] Sushila, J. Singh, and Y. S. Shishodia, "An efficient analytical approach for MHD viscous flow over a stretching sheet via homotopy perturbation sumudu transform method," Ain Shams Engineering Journal, vol. 4, no. 3, pp. 549-555, 2013.

[6] B. Raftari, S. T. Mohyud-Din, and A. Yildirim, "Solution to the MHD flow over a non-linear stretching sheet by homotopy perturbation method," Science China: Physics, Mechanics and Astronomy, vol. 54, no. 2, pp. 342-345, 2011.

[7] A. Chakrabarti and A. S. Gupta, "Hydromagnetic flow and heat transfer over a stretching sheet," Quarterly of Applied Mathematics, vol. 37, no. 1, pp. 73-78, 1979.

[8] H. I. Andersson, "An exact solution of the Navier-Stokes equations for magnetohydrodynamic flow," Acta Mechanica, vol. 113, no. 1-4, pp. 241-244, 1995.

[9] P. S. Gupta and A. S. Gupta, "Heat and mass transfer on a stretching sheet with suction and blowing," The Canadian Journal of Chemical Engineering, vol. 55, no. 6, pp. 744-746, 1977.

[10] L. J. Crane, "Flow past a stretching plate," Journal of Applied Mathematics and Physics, vol. 21, no. 4, pp. 645-647, 1970.

[11] J.-H. He, "Addendum: new interpretation of homotopy perturbation method," International Journal of Modern Physics B, vol. 20, no. 18, pp. 2561-2568, 2006.

[12] H. B. Keller, "Accurate difference methods for linear ordinary differential systems subject to linear constraints," SIAM Journal on Numerical Analysis, vol. 6, pp. 8-30, 1969. 


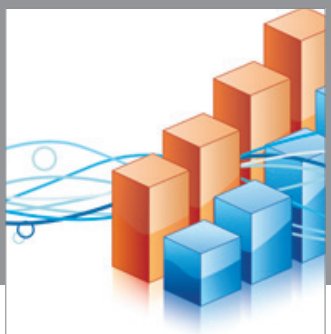

Advances in

Operations Research

vatem alat4

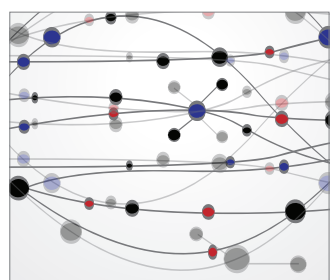

\section{The Scientific} World Journal
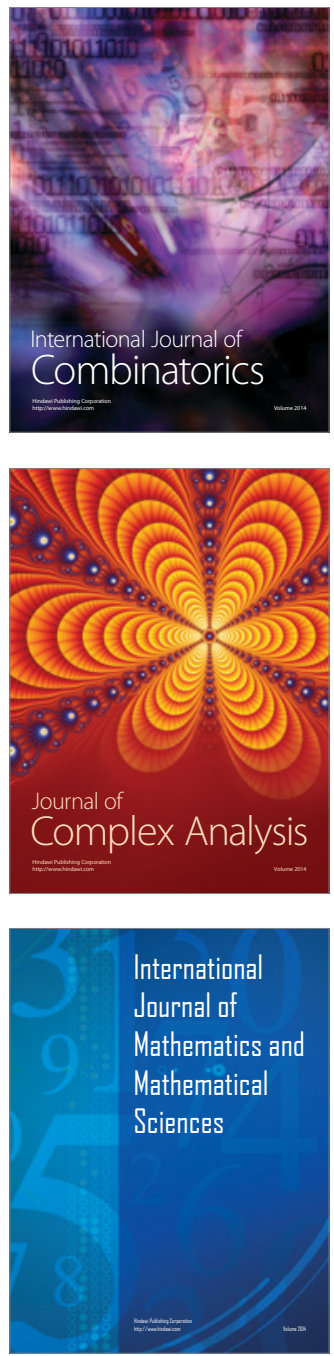
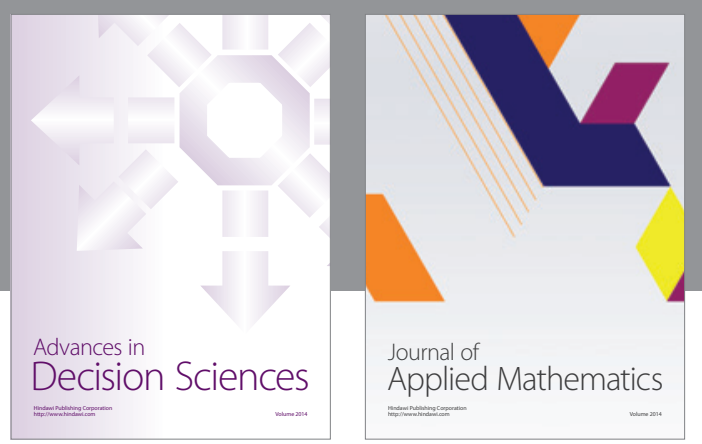

Algebra

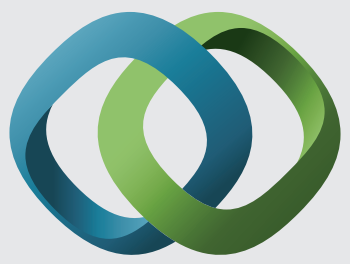

\section{Hindawi}

Submit your manuscripts at

http://www.hindawi.com
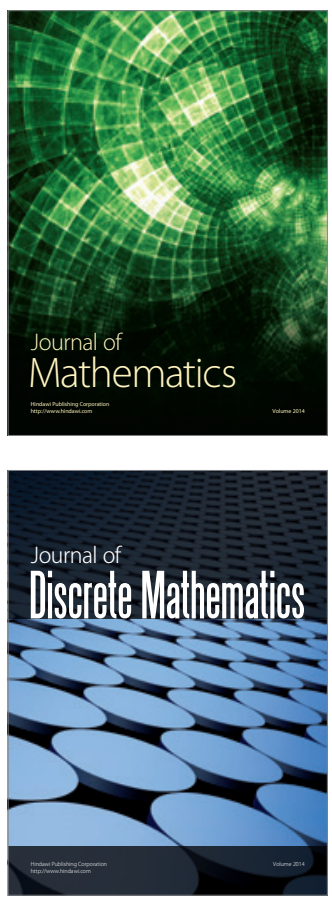

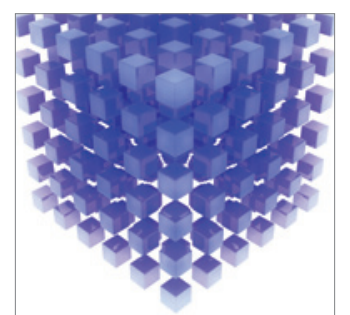

Mathematical Problems in Engineering
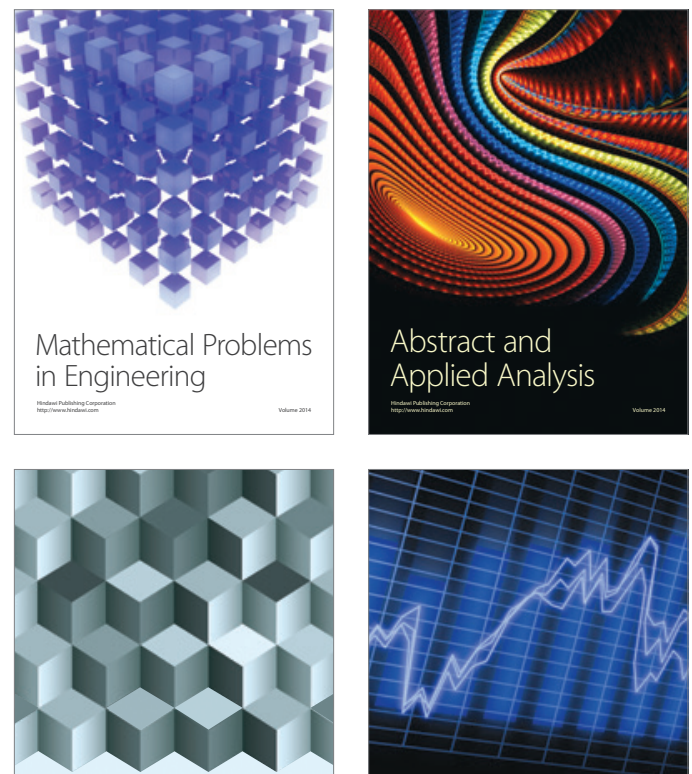

Journal of

Function Spaces

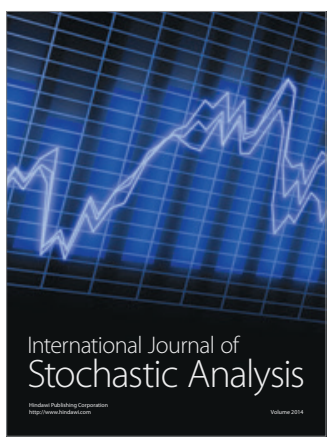

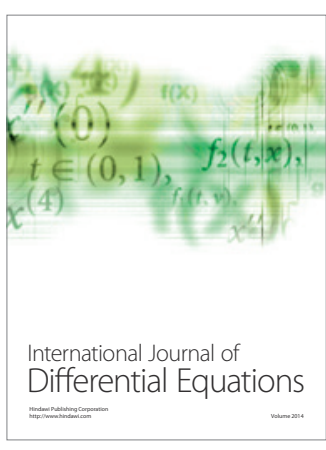
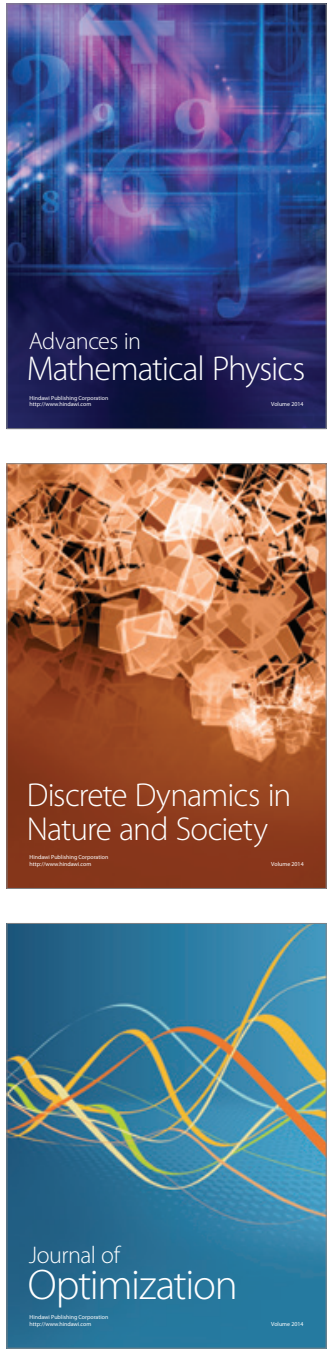